\title{
Camino Verde (The Green Way): evidence- based community mobilisation for dengue control in Nicaragua and Mexico: feasibility study and study protocol for a randomised controlled trial
}

\author{
Neil Andersson ${ }^{1,2^{*}}$, Jorge Arostegui ${ }^{3}$, Elizabeth Nava-Aguilera', Eva Harris ${ }^{4}$ and Robert J Ledogar ${ }^{5}$
}

From The Camino Verde Trial colloquium

Acapulco, Mexico. 17-21 June 2013

\begin{abstract}
Background: Since the Aedes aegypti mosquitoes that transmit dengue virus can breed in clean water, WHO-endorsed vector control strategies place sachets of organophosphate pesticide, temephos (Abate), in household water storage containers. These and other pesticide-dependent approaches have failed to curb the spread of dengue and multiple dengue virus serotypes continue to spread throughout tropical and subtropical regions worldwide. A feasibility study in Managua, Nicaragua, generated instruments, intervention protocols, training schedules and impact assessment tools for a cluster randomised controlled trial of community-based approaches to vector control comprising an alternative strategy for dengue prevention and control in Nicaragua and Mexico.

Methods/Design: The Camino Verde (Green Way) is a pragmatic parallel group trial of pesticide-free dengue vector control, adding effectiveness to the standard government dengue control. A random sample from the most recent census in three coastal regions of Guerrero state in Mexico will generate 90 study clusters and the equivalent sampling frame in Managua, Nicaragua will generate 60 clusters, making a total of 150 clusters each of 137-140 households. After a baseline study, computer-driven randomisation will allocate to intervention one half of the sites, stratified by country, evidence of recent dengue virus infection in children aged 3-9 years and, in Nicaragua, level of community organisation. Following a common evidence-based education protocol, each cluster will develop and implement its own collective interventions including house-to-house visits, school-based programmes and inter-community visits. After 18 months, a follow-up study will compare dengue history, serological evidence of recent dengue virus infection (via measurement of anti-dengue virus antibodies in saliva samples) and entomological indices between intervention and control sites.
\end{abstract}

Discussion: Our hypothesis is that informed community mobilisation adds effectiveness in controlling dengue.

Trial registration: ISRCTN27581154.

\footnotetext{
* Correspondence: andersson@ciet.org

${ }^{1}$ Centro de Investigación de Enfermedades Tropicales (CIET), Universidad

Autónoma de Guerrero, Acapulco, Mexico

${ }^{2}$ Department of Family Medicine, McGill University, Montreal, Canada

Full list of author information is available at the end of the article
} 


\section{Background}

Recognition of dengue as a major health issue is increasing, especially in Asia and Central and South America [1]. In Nicaragua and Mexico, as in most dengue endemic countries, the national pesticidedependent approaches have failed to curb the spread of this mosquito-borne disease. Several reviews have shown pace-spraying with pesticide to kill adult mosquitoes to be of little value [2]. Multiple serotypes of dengue virus continue to move northward through Latin America.

Failure to curb the dengue epidemic has led to resurgence of interest in community mobilisation for its vector control. A 2007 systematic review of community-based dengue control programmes, including two randomised controlled trials, found only weak evidence "that community-based dengue control programmes alone and in combination with other control activities can enhance the effectiveness of dengue control programmes" [3]. However, none of these earlier studies used clustered designs.

Several cluster trials have since shown an impact on vector densities. A team in Cuba published a trial of community mobilisation in 16 clusters compared with 16 controls, using vector breeding indices as the outcome [4]. In India, community-level provision of water container covers, clean-up campaigns, and dissemination of dengue information through schoolchildren also reduced vector density [5]. In Thailand, community volunteers reduced vector density [6]. No impact could be detected in a comparison of two communities in the Philippines [7].

A 2011 systematic review of 22 studies involving education messages for community-based dengue prevention concluded that these were effective in reducing entomological indices, but none measured dengue occurrence [8]. A recent systematic review of 14 studies of Bacillus thuringiensis israelensis found ample evidence that this reduced the number of Aedes aegypti breeding forms but only one study provided any evidence of impact on dengue risk; the study reported one dengue case in the intervention area and 15 cases in the control area [9].

The literature suggests that non-pesticide measures should prevent dengue, but there is very little direct evidence of this. A pesticide-free alternative has potential health, economic and environmental benefits.

\section{Feasibility study in Managua 2004-2008 Eligible participants}

In Managua, Nicaragua, 10 intervention and 20 control sites (132 houses per site, 3,960 households and 3,300 children aged 3-9 years) piloted development of instruments and protocols for a pesticide-free intervention.

\section{Willingness of participants to be randomised}

There was no allocation concealment or randomisation to intervention and control. The feasibility study focussed on community intervention dynamics and development of impact assessment instruments.

\section{Interventions}

Baseline survey teams collected serological, entomological and behavioural evidence during biannual measurement cycles. Community volunteers used this evidence to promote dialogue on the issue of dengue prevention and to develop interventions in 10 sites. Interventions included household visits, active demonstration of mosquito eggs and larvae/pupae in the home, and use of simple tools and procedures for breeding site elimination.

\section{Follow-up and response rates to questionnaires}

Of 5596 households contacted in 2008, all allowed examination of vector reservoirs outside the building; all but 21 answered questions about recent illness in the household (99.7\%).

\section{The proposed outcome measures}

Planned outcomes were serological IgG ELISA analysis of paired saliva samples to detect a decrease in the serological evidence of recent dengue virus infection in children aged 3-9 years; entomological indices; and household measures of social capital. Evaluation teams measured entomological, immunological and behavioural indicators each year. Covariates included use of temephos, type of water container, other containers near the dwelling, education of household head, involvement in other community matters, and number of people in the household.

\section{Standard deviation of the outcome measure, to estimate sample size}

In 2004-8, incidence of dengue virus infection in children over the two measurements ranged between $1.5 \%$ and $7.4 \%$ in the control communities; the reporting rate of dengue varied between $0.6 \%(1 / 166)$ and 5.3\% (10/189); between $11 \%(20 / 183)$ and 50\% (94/188) households were positive for Aedes aegypti larvae and/or pupae.

\section{Adherence/compliance rates}

The total 3,217 households with children aged 3-9 years yielded 2,992 saliva samples from these children $(7 \%$ or 225 declined or were not present at the time of the visit). By the fourth year of the feasibility study (2007), twice as many households in the intervention sites (15.5\%) as in the control sites $(7.1 \%)$ said they participated directly in community dengue control exercises, and $50.7 \%$ in 
intervention and $39.5 \%$ in control sites reported having looked for larvae in their water stores over the last week.

\section{Intraclass correlation coefficient (ICC)}

Using an ANOVA framework for the 20 control communities in 2007, we estimated an ICC of 0.78 for the saliva samples of 3-9 year-old children and 0.396 for households positive for Aedes aegypti larvae and/or pupae.

\section{Comment}

As with many studies of this type [10], the feasibility study in Nicaragua was not randomised. We therefore did not evaluate the impact on dengue but estimated parameters needed to design the main study. The Nicaraguan study fine-tuned a dengue-specific intervention strategy called "socialising of evidence for participatory action" (SEPA). The study demonstrated feasibility of Camino Verde in vector control, with likely gains for the intervention communities [11-14]. The long history of community mobilisation in Nicaragua raises issues of replicability in other contexts. We therefore included southern Mexico in the main trial.

\section{Objectives of the Camino Verde trial}

The research question is: Among residents of Managua and the State of Guerrero in Mexico, does evidence-based community mobilisation add effectiveness to the usual dengue control strategies to decrease the risk of dengue virus infection using a parallel group cluster RCT?

The Camino Verde hypothesis is that informed community mobilisation adds effectiveness in controlling dengue. The trial is concerned with the real-life effect of the intervention in the coastal region of Guerrero state and in Managua, rather than its effectiveness under ideal conditions $[15,16]$.

The overall objective is to reduce dengue risk through evidence-based knowledge translation. An explicit objective is to develop a sustainable community-oriented vector control approach that reduces the need for pesticides in water used in or around homes or areas that children frequent, or likely to be used with food or even as drinking water.

Specific objectives are (1) Determine entomological and serological status of the virus-vector-host ecosystem as well as knowledge, attitudes and behaviour underlying community-led interventions; (2) In partnership with intervention communities, translate this knowledge on dengue vector behaviour and immunology into household and community action against dengue; (3) Identify the impact of informed community mobilisation on dengue risk and its added effectiveness regarding other infections, social capital and longer term economic welfare.

\section{Methods/Design \\ Design of the study}

This parallel group pragmatic cluster randomised controlled trial will test informed community mobilisation in dengue control, measured by risk of dengue virus infection, occurrence of self-reported dengue cases, and vector density. The baseline will include a household questionnaire, entomological assessment and saliva samples from children aged 3-9 years. After the baseline survey, a sampling statistician not associated with the trial will randomise the 150 sites to intervention or control, stratified by area, entomological indices and serological evidence of recent dengue virus infection in children. Interventions will be based on evidence of Aedes aegypti infestation, explanation of the dengue vector life cycle, and information on how to prevent larvae and pupae from becoming adult mosquitoes. Different ways of transmitting this knowledge and acting upon it will be designed and implemented by the participating communities. The intervention will run for at least a full year. A multi-method impact survey will cover all 150 clusters with no in-cluster sampling. This will measure risk of dengue virus infection, disease reports, and entomological indices of the vector Aedes aegypti. Figure 1 is a flow-diagram of the trial.

\section{The setting}

Nicaragua reports around 10,000 cases of dengue each year; all four serotypes circulate, with one serotype dominating in each season [17]. Between 2003 and 2007, registered dengue cases in Mexico increased 10-fold to 48,456 . Guerrero now reports a high rate of dengue haemorrhagic fever $(35 / 100,000)$, second in the country only to Quintana Roo, with all four serotypes well established. The Nicaraguan and Mexican government strategy for control of the Aedes aegypti mosquito rests on insertion of a packet of temephos (trade name Abate) in household water containers, known in both countries as "abatización".

Participants are residents in a random sample of 150 clusters (100-120 households each) from the latest census in each country. In Mexico, a stratified last-stage-random sample generated 90 enumeration areas, 30 representing each of three regions (Costa Grande, Acapulco and Costa Chica regions of Guerrero state). In Nicaragua, 60 enumeration areas represent of the capital city, Managua, where approximately onefourth of the Nicaraguan population resides.

\section{Interventions}

"Socialising of evidence for participatory action" (SEPA) (http://www.ciet.org/en/method/sepa-communication/), was fine-tuned during the Managua feasibility study. Although there will be a high degree of local 


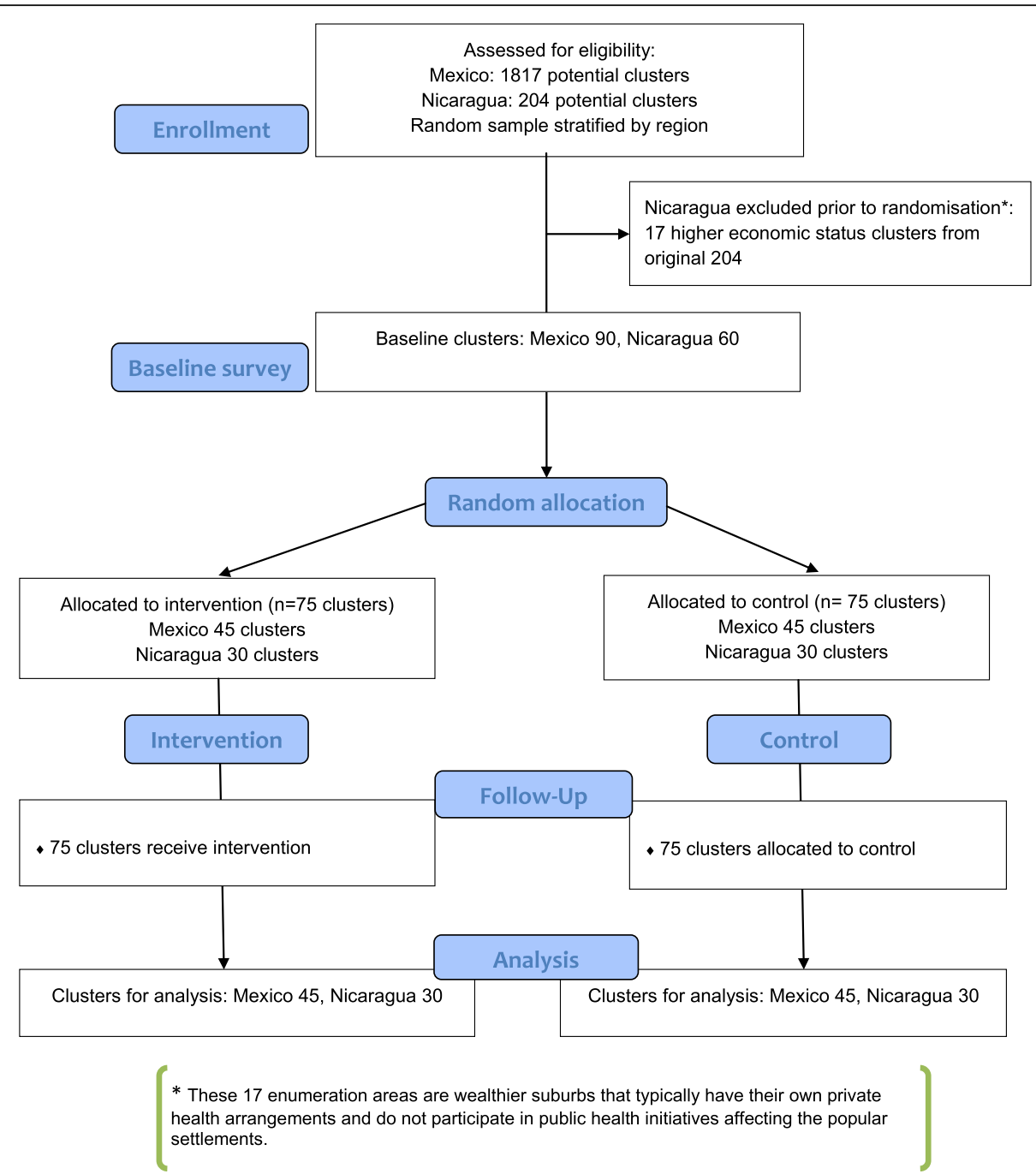

Fig. 1 Camino Verde participant flow diagram

customising of the intervention, all sites will follow the same protocol to generate community-wide activities that vary widely in character and scope but all built around information about the life cycle of the mosquito and how to interrupt it:

1. Feedback of evidence from the baseline survey to community representatives in discussion groups to author the locally-defined interventions;

2. Brigadistas or volunteers from the communities will serve as organisers and as educators - trained by facilitators from the research team, who will then be withdrawn from the communities - conducting visits to schools and household (visitas de acompañamiento) to show the evidence of infestation in water receptacles;

3. Structural interventions will change the Aedes aegypti ecosystem in each intervention community;
4. Intercommunity visits and peer evaluations will share experiences between communities and strengthen the group dynamics and the collective preventive action.

In both intervention and control enumeration areas, interviewers from the baseline survey will return individual serology results and explanations of their interpretation to households where 3-9 year old children provided saliva samples. Other activities will depend on community preferences and, based on the feasibility study and discussions with pilot communities, could include neighbourhood clean-up campaigns, puppet theatre, basketball competitions, rap (reguetón) and other songs about dengue, parades, and poster competitions.

Community-to-community peer monitoring is an anticipated feature of the intervention, to be customised 
to suit the different conditions in the two countries. In Nicaragua, the four-year feasibility study has already provided a good idea of the change process so their emphasis may be more on the quantitative data. Both Mexican and Nicaraguan peer process evaluations will involve members of the research team from one cluster or region visiting another cluster or region and gathering both quantitative and qualitative information to provide project management and community leaders with actionable details about the intervention process, to inform any necessary mid-course corrections. In both countries, the inter-community visits and process evaluation will provide encouragement and training for intervention field staff and communities alike.

Quantitative information will include data on the last time a member of the cluster's own research team visited the household and data on larval/pupal infestation as found by the monitoring team. Qualitative information will include recommendations from householders for improvement in the programme and responses to questions about the most significant change that community members have observed since the intervention began [18]. With the focus on the nature of the change, purposive sampling will yield a number of accounts to be processed [19].

\section{Outcomes}

The main outcome measures are (i) risk of dengue virus infection as measured by a minimum of two-fold increase in anti-dengue virus IgG antibodies across paired saliva samples collected before and after the 2012 dengue season in children aged 3-9 years [20] (ii) dengue cases (any age) in the last year reported by the household respondent; and (iii) entomological indices of Aedes aegypti breeding sites, using the protocol developed in the Nicaragua feasibility study [21].

\section{Serology samples}

Interviewers who have not been part of the intervention will collect saliva samples from children (estimating 140 households in each cluster, one child aged 3-9 years in every three households) in both intervention and control clusters at the beginning and at the end of the dengue season. During household visits, the interviewer will ask children to spit $0.5-2 \mathrm{ml}$ of saliva into a plastic receptacle. Returning fieldworkers will transport samples to the CIET laboratories in Acapulco and the Ministry of Health National Virology Laboratory in Managua, divided into labelled aliquots and stored at $-80{ }^{\circ} \mathrm{C}$ until processing. We will process the pre- and post-season samples side-byside by capture ELISA and will consider an increase in IgG antibody units of greater than or equal to two-fold indicative of increased dengue virus infection risk. The analysis will be repeated for three-fold and four-fold changes.

The serology protocol requires incubation of polystyrene plates fixed with 100ul anti-human IgG antibodies (Sigma) incubated for $24 \mathrm{~h}$, then incubated at $37{ }^{\circ} \mathrm{C}$ for one hour with $50 \mu \mathrm{l}$ of undiluted saliva, followed by incubation for $1 \mathrm{~h}$ with $50 \mu \mathrm{l}$ of antigen prepared from all four dengue serotypes prepared in-house in Nicaragua from infected mouse brain, with four washes in between each step. We will measure the colour change after adding $50 \mu \mathrm{l}$ of antidengue virus immunoglobulin conjugated to horseradish peroxidase (prepared and titrated in Nicaragua) followed by $50 \mu \mathrm{l}$ of TMB substrate (Sigma) at $450 \mathrm{~nm}$ in an ELISA reader. We will calculate antibody units as $=[(\mathrm{OD}$ sample - OD negative control)/(OD positive control - OD negative control)] $\times 100$.

\section{Breeding site assessment}

Entomology evaluators who are unaware of the intervention status of sites will inspect indoor and outdoor containers, including natural habitats such as tree holes and leaf axils, that might harbour Aedes aegypti or Aedes albopictus immature forms (larvae or pupae). They will examine containers in dark or shaded areas using flash lights. Although recent research questions the value of Aedes aegypti larvae as a predictor of adult mosquito females in households [22-24], we include larval indices as both Mexican and Nicaraguan Ministries of Health rely on these for dengue mosquito surveillance [25]. Evaluators will examine all container types (tires, flower vases, water storage barrels, laundry wash basins, plastic tarps, puddles, and discarded containers (bottles, cans, drums, metal pots, plastic containers) [26]. They will collect all the larvae and pupae from each positive container using nets and pipettes, place them in labelled and capped plastic vials, and send them to the National Entomology Reference Laboratory in Nicaragua and CIET Entomology Laboratory in Mexico for identification. Double data entry with discrepancy verification will reduce keystroke errors before linking data with other study databases.

\section{Entomology indices}

These are a) House Index (HI), number of houses with one or more containers positive for immature Aedes aegypti divided by the number of houses sampled multiplied by 100; b) Container Index (CI) number of water-holding containers with immature Aedes aegypti divided by the number of water-holding containers inspected multiplied by 100; c) Breteau index (BI) number of water-holding containers with immature Aedes aegypti per every 100 households inspected; d) pupae per container or household 
inspected; and e) pupae per person (pu/per) number of pupae collected over the total number of inhabitants of the households inspected.

Secondary outcomes focus on the social capital gained from community engagement and the partial outcomes toward acceptance of the Camino Verde, including knowledge of and attitude to prevention, intention to change prevention behaviour, agency (collective and selfefficacy), discussion/socialisation, and prevention-related action. Additional secondary outcomes include purchase and use of pesticide, and reduced expenditure on health care as a consequence of dengue virus infection.

\section{Type of analysis}

\section{Data entry and security}

The household responses to the questionnaires will be entered twice by independent operators who are ignorant of intervention status of the sites, with verification of discordant entries from the original questionnaires. A data manager ignorant of intervention status of the sites will check digitised data for logical errors. Questionnaires from intervention and control sites will be handled in exactly the same way, with all data technicians unaware of the intervention status of clusters. Site identities will be masked before analysis.

\section{Principal analysis}

With 150 clusters allocated evenly between intervention and control arms, the cluster analysis of primary outcomes will rest on a t-test, following an intention-totreat principle (everyone included in each cluster, per allocation). The simultaneous evaluation in control and intervention sites will account for temporal effects. Serological status, reports of dengue illness, and vector indices lend themselves to analysis as continuous variables. Recent infection in children, adopting a cut-off of a two-fold increase of IgG units between samples, and pupae-positive households, are both dichotomized variables; the percent of households with serological evidence of recent infection in any child or with larvae/ pupae-positive entomology serves as a continuous variable in each site. We will report outcomes as absolute event rates among intervention and control groups, with relative risk reduction (RRR) and 95\% confidence intervals.

\section{Secondary analysis}

We will use individual-level data allowing for informative clustering in a non-linear mixed model with a robust variance and non-fixed odds-ratio [27] or generalised linear mixed model (GLMM) assuming cluster as a random effect [28]. We propose to examine average individual effects (for example, children with increased serological evidence of recent dengue virus infection, recent self-reported cases of dengue, or entomological parameters) while accounting for temephos exposure and water supply.

Sensitivity analysis will focus on gender-specific responses and exclusion of sites with prominent government pesticide intervention.

Planned subgroup analysis will focus on regional variations of the protective effect of Camino Verde, especially relating to population density (urban/rural) and the particular choices of intervention.

\section{Missing data}

All communities experience in-migration and outmigration. We will add new arrivals to the study but will not follow those leaving the household clusters. There is no reason to expect differential out-migration between intervention and control clusters. Self-selection (decision not to participate or not to answer certain questions) is a concern, given the structured engagement implied by the intervention. Those who opt not to respond may be less involved with dengue control - thus reducing the measured effect. We will analyse missing data using Amelia II [29] to impute values for missing data with an EM algorithm for the primary outcome (serological evidence of recent dengue virus infection). Estimates will reconcile data from ten imputed data sets using Rubin's approach [30] in the R package Zelig [31].

\section{Economic analysis}

The sample size estimate for this trial uses relatively rare primary outcomes, so it will be adequately powered to consider economic implications of the intervention, which could be profound. Informed community mobilisation could reduce dengue rates directly but, by increasing social engagement, it could also increase the proportion of the population enabled to benefit from other prevention interventions, increasing their impact correspondingly. The dengue virus infection end-point (serology) is most relevant for economic analysis of the direct impact, although reduction of entomological indices also has resonance. By costing the intervention, it should be possible to document the costs of dengue cases avoided [32]. Documenting the real cost of preventing dengue will be less simple. A cost-benefit approach will be relevant, with benefits identified and qualified by communities themselves. Individuals who opt to reduce their own dengue risk probably do so based on perceived costs and benefits of prevention we have called this the individual "cost benefit equation" [33]. This equation can lead to very different decisions from those imagined by dengue control programmes, especially in the context of abatización, which removes choice and changes the way people estimate their own value in the choices they make. 


\section{Implications for health services}

The case to be made is that informed community mobilisation reduces dengue risks, without reliance on imported pesticide. A secondary case is that the costs compare favourably with the gains of doing this; pesticide-free prevention requires less treatment of cases, less work time lost, and less importation of expensive pesticides.

\section{Software}

CIETmap [34] is a hybrid vector-raster GIS software with seamless linkages between epidemiological analysis and mapping. The package provides a windows-like interface with the popular open-source statistical programming language $\mathrm{R}$.

\section{Power calculation}

Data from the feasibility study in Managua facilitated sample size estimation. Using the dengue serology results as the principal outcome, control communities in 2004-8 showed a range of $1.5 \%$ to $7.4 \%$ (k-statistic 0.328). Follow-up of 40 children in each of 150 clusters (75 clusters per arm) would detect a $33.3 \%$ reduction in incidence $(4.5 \%$ to $3 \%)$ with $80 \%$ power at a significance level of $5 \%$. These calculations assume no interaction effects and a two-sided test with cluster as unit of primary analysis in unmatched parallel groups. Calculations used a spreadsheet based on the trial simulator devised by Taylor and colleagues [35].

\section{Randomisation \\ Allocation concealment}

Randomisation will be conducted centrally by a sampling statistician who is not engaged in the rest of the study. Computer-generated random numbers will determine intervention status in each stratum, defined by the baseline study. The intervention will begin promptly after randomisation with disclosure of intervention status at that time.

\section{Blinding}

The prevention efforts will be obvious to residents in the intervention sites, and some outcomes (particularly conscious knowledge) could be influenced by knowledge of intervention status. The main outcome indicators (reduction in serological evidence of recent dengue virus infection or entomological indices) would be less susceptible to this bias. To prevent potential bias, interviewers should ideally be unaware if they are interviewing in intervention or control clusters. Data managers will be unaware of intervention status of clusters.

\section{Contamination}

There may be some contamination of the control clusters: through people interacting between the clusters at the individual or household level; through schools (children from control clusters going to schools in the intervention clusters); and through local government or NGOs taking up the emerging evidence to guide interventions in control clusters. Perhaps a bigger concern, in urban areas, is that mosquitoes from neighbouring households influence rates in the intervention sites and that children may get bitten by infected mosquitoes outside of their intervention site [36,37]. Both types of contamination will reduce the measured difference between control and intervention clusters. We cannot avoid this effect. We will document spread of the intervention beyond the sites in several regards: (i) mechanisms for generalising evidence, (ii) uptake at policy level and in the public discourse, (iii) new initiatives or changes in approach by agencies involved in dengue control, and (iv) impact in the control clusters as well as in the intervention clusters.

\section{Ethical considerations \\ Ethical review}

The Institutional Review Board (IRB) at the University of California, Berkeley, and the CIET IRB reviewed the Nicaraguan arm. The Research Ethics Board of CIETcanada and the Ethics Committee of the Centro de Investigación de Enfermedades Tropicales at the Universidad Autónoma de Guerrero reviewed the Mexican arm.

1. Informed consent: Using a standard script, in the household survey, interviewers explain to respondents the nature of the survey, its voluntary character, and the anonymous quality of the process. They explain that respondents may decline to answer any questions they do not wish to, and may terminate the interview at any time. They then ask respondents for their consent to conduct the interview.

2. Samples: Separate informed consent is obtained from a parent or guardian for collecting the saliva samples with assurance that the results will be returned to them. To allow linkage of the repeat sample result with individual risk factors from the questionnaire responses, we use adhesive labels with ID codes fixed to the sample, to the completed questionnaire and, in the case of Nicaragua, to an administrative sheet that includes the date and time of the sample.

3. Ensuring confidentiality: Training of fieldworkers and data operators will emphasize responsibility for maintaining confidentiality of all information to which they have access during the work. We will 
report only grouped findings, in a way that does not allow identification of any individuals or individual communities. In the household survey, no names or identifiers will be recorded next to individual responses. Unique number labels will be used to link saliva specimens to questionnaire responses, without identifying the person by name. In individual followup for repeat samples, we will record the name of the child in order to match this from visit to visit and to be able to deliver results of the tests to parents or guardians. In focus groups, no names of focus group participants will be recorded, and reports of focus group findings will not identify individual communities.

4. Protection of emotional well-being: We do not expect the questions to awaken distressing memories. To the contrary, we expect the opportunity to engage in household and community protection to be uplifting and self-affirming.

5. Normative pressure within communities: Both the CIETinternational and CIETcanada ethical review boards raised questions regarding possible stigmatising of residents who failed to participate in community vector control activities. Both boards were satisfied by the responses they received from the field teams on this subject. We will prepare a full discussion of the ethical issues and our position regarding them and submit it for publication in a peer-reviewed journal.

6. Security of data: Digital records will be secure and accessible only to the lead epidemiologist. Original paper records will be securely transported, stored, retained and finally destroyed in accordance with CIET guidelines for security, storage and eventual destruction of paper records [38].

7. Promotion of equal opportunities: At least one half of field workers in the interview teams and the local action teams will be women. The field team leader in Mexico, Elizabeth Nava, is a member of the CIET academic council. Eva Harris is the nominated principal investigator of the Nicaragua team.

\section{Discussion}

Current dengue control measures in Latin America rest on an organophosphate pesticide, temephos (Abate), inserted in the household water storage containers that are often breeding sites for Aedes aegypti, the main vector for dengue virus in the region. Many national governments have used temephos in household water stores. The WHO approval to do so $[39,40]$ cites the lack of evidence of toxicity.

Temephos is not licensed for human consumption in the USA [41]; the US Environmental Protection Agency says temephos "...has no food uses, is not likely to be found in drinking water, and is not used in or around homes or areas that children frequent" [42]. Studies not sponsored by the producers have documented animal toxicity [43, 44] and genotoxicity [45]. Resistance of the mosquito to this pesticide is already well documented and is a growing concern, given the variable coverage and consistency of many government-initiated programmes using temephos for dengue control [46-52]. We could not identify any community-based randomised controlled trials showing a protective effect of temephos against dengue virus infection.

Given the current government commitment to temephos, it will take a while to generate full participation of state and local authorities. Assuming the intervention is successful, there is an ethical obligation to implement it also in control communities. As this is unlikely within the 3-year time period of this project, other funds will be sought for that purpose.

There is also a strong community-building component integrated into the trial design; one of the goals is to measure the impact of the intervention on social capital. In each intervention cluster, the community action team will be trained in evidence-based communication as well as in ways of engaging with residents that are fully respectful of personal and community autonomy.

\section{Acknowledgements}

Funding

The UBS Optimus Foundation provided funding for the feasibility study in Nicaragua, the full trial in Nicaragua and Mexico, and publication of this article.

Availability of data and materials

No further data available.

\section{Authors' contributions}

NA designed the study, wrote the proposals, and prepared this article; JA is lead researcher in the Nicaragua feasibility study, and contributed to the design and drafting of this protocol; ENA is lead researcher in Mexico and contributed to the design and drafting; EH was the principal investigator in the Nicaraguan feasibility study and current trial, and contributed to design and drafting of this protocol and to writing the proposals; RJL contributed to the design and drafting of the protocol.All authors read and approved the final manuscript.

\section{Competing interests}

The authors declare that they have no competing interests.

Consent for publication

Not applicable.

\section{Ethics approval and consent to participate}

The Institutional Review Board (IRB) at the University of California, Berkeley, and the CIET IRB reviewed and approved the Nicaraguan arm. The Research Ethics Board of CIETcanada and the Ethics Committee of the Centro de Investigación de Enfermedades Tropicales at the Universidad Autónoma de Guerrero reviewed and approved the Mexican arm.

\section{About this supplement}

This article has been published as part of BMC Public Health Volume 17 Supplement 1, 2017: The Green Way to Aedes aegypti mosquito control: aspects and implications of the Camino Verde trial in Mexico and Nicaragua. The full contents of the supplement are available online at https:// bmcpublichealth.biomedcentral.com/articles/supplements/volume-17supplement-1. 


\section{Publisher's Note}

Springer Nature remains neutral with regard to jurisdictional claims in published maps and institutional affiliations.

\section{Author details}

${ }^{1}$ Centro de Investigación de Enfermedades Tropicales (CIET), Universidad Autónoma de Guerrero, Acapulco, Mexico. ${ }^{2}$ Department of Family Medicine, McGill University, Montreal, Canada. ${ }^{3} \mathrm{CIET}$, Managua, Nicaragua. ${ }^{4}$ Division of Infectious Diseases and Vaccinology, School of Public Health, University of California, Berkeley, CA, USA. ${ }^{5}$ CIET international, New York, NY, USA.

\section{Published: 30 May 2017}

\section{References}

1. Brady OJ, Gething PW, Bhatt S, Messina JP, Brownstein JS, Hoen AG, et al. Refining the global spatial limits of dengue virus transmission by evidencebased consensus. PLoS Negl Trop Dis. 2012;6:e1760.

2. Esu E, Lenhart A, Smith L, Horstick O. Effectiveness of peridomestic space spraying with insecticide on dengue transmission; systematic review. Tropical Med Int Health. 2010;15:619-31.

3. Heintzea C, Velasco Garridob M, Kroeger A. What do community-based dengue control programmes achieve? A systematic review of published evaluations. Trans R Soc Trop Med Hyg. 2007;101:317-25.

4. Vanlerberghe V, Toledo ME, Rodríguez M, Fomez D, Baly A, Benitez JR, et al. Community involvement in dengue vector control: cluster randomised trial. BMJ. 2009;338:1959.

5. Arunachalam N, Tyagi BK, Samuel M, Krishnamoorthi R, Manavalan R, Tewari SC, et al. Community-based control of Aedes aegypti by adoption of eco-health methods in Chennai City, India. Pathog Glob Health. 2012;106:488-96.

6. Kittayapong $\mathrm{P}$, Thongyuan S, Olanratmanee P, Aumchareoun W, Koyadun S, Kittayapong $\mathrm{R}$, et al. Application of eco-friendly tools and eco-bio-social strategies to control dengue vectors in urban and peri-urban settings in Thailand. Pathog Glob Health. 2012;106(8):446-54.

7. Fe E. Community-based dengue vector control: experiences in behavior change in Metropolitan Manila. Philippines Pathog Glob Health. 2012;106: 455-60

8. Al-Muhandis N, Hunter PR. The value of educational messages embedded in a community-based approach to combat dengue fever: a systematic review and meta regression analysis. PLoS Negl Trop Dis. 2011;5:e1278.

9. Boyce R, Lenhart A, Kroeger A, Velayudhan R, Roberts B, Horstick O. Bacillus thuringiensis israelensis (Bti) for the control of dengue vectors: systematic literature review. Tropical Med Int Health. 2013;18:564-77.

10. Arain M, Campbell MJ, Cooper CL, Lancasteret GA. What is a pilot or feasibility study? A review of current practice and editorial policy. BMC Med Res Methodol. 2010;10:67.

11. Arostegui J, Carcamo C, Hammond SN, Coloma J, Balmaseda A, Andersson $\mathrm{N}$, et al. Evidence-based community-derived interventions for the control of the dengue virus vector Aedes aegypti in Managua Nicaragua. $54^{\text {th }}$ Annual Meeting of the American Society of Tropical Medicine and Hygiene, Washington, DC; 2005

12. Arostegui J, Hammond SN, Carcamo C, Suazo H, Coloma J, Balmaseda A, et al. Impact of evidence-based community-derived interventions for the control of the dengue virus vector Aedes aegypti in Managua, Nicaragua. $55^{\text {th }}$ Annual Meeting of the American Society of Tropical Medicine and Hygiene, Atlanta; 2006.

13. Arostegui J, Suazo H, Carcamo C, Hernandez C, Coloma J, Balmaseda A et al. Sustained impact of evidence-based community-derived communication strategies for the control of the dengue virus vector Aedes aegypti in Managua, Nicaragua. 56th Annual Meeting of the American Society of Tropical Medicine and Hygiene, Philadelphia; 2007.

14. Arostegui J, Suazo H, Coloma J, Carcamo C, Hernandez C, Balmaseda A, et al. Impact on serological, entomological, and behavioral indices of an evidence-based community-derived communication programme for the control of Aedes aegypti and dengue in Managua, Nicaragua. $57^{\text {th }}$ Annual Meeting of the American Society of Tropical Medicine and Hygiene, New Orleans; 2008.

15. Califf RM, Woodlief LH. Pragmatic and mechanistic trials. Eur Heart J. 1997;9:367-70.

16. MacPherson H. Pragmatic Trials Complementary Therapies in Medicine. 2004;12:136-40
17. Ministerio de Salud.MINSA. Epidemiology Directorate Reports (1990-1996, 1997-2001, and 2002-2006). Managua, Nicaragua.

18. Dart J, Davies R. A dialogical, story-based evaluation tool. Am J Eval. 2003;24:137-55

19. Weinstein M. TAMS Analyzer. 2012 Available from: http://tamsys.sourceforge. net. Accessed 1 May 2017.

20. Balmaseda A, Saborio S, Tellez Y, Mercado JC, Pérez L, Hammond SN, et al. Evaluation of immunological markers in serum, filter-paper bloodspots, and saliva for dengue diagnosis and epidemiological studies. J Clin Virol. 2008; 43:287-91.

21. Hammond SN, Lugo E, Moreno G, Gordon A, Kuan G, Delgado M, et al. Characterization of Aedes aegypti (Diptera: Culcidae) breeding sites in urban Nicaragua: Tools for developing and assessing vector control strategies. J Med Entomol. 2007:44:851-60.

22. Focks DA, Chadee DD. Pupal survey: an epidemiologically significant surveillance method for Aedes aegypti: an example using data from Trinidad. Am J Trop Med Hyg. 1997:56:159-67.

23. Barrera R, Amador M, Clark GG. Use of the pupal survey technique for measuring Aedes aegypti (Diptera: Culicidae) productivity in Puerto Rico. Am J Trop Med Hyg. 2006;74:290-302.

24. Morrison A, Gray CK, Getis A, Astete H, Sihuincha M, Focks D, et al. Temporal and geographic patterns of Aedes aegypti (Diptera: Culicidae) production in Iquitos, Peru. J Med Entomol. 2004;41:1123-42.

25. Pan American Health Organization. Dengue and dengue hemorrhagic fever in the Americas: guidelines for prevention and control. Scientific Publication \#548, 1994.

26. Hammond SN, Gordon AL, Lugo Edel C, Moreno G, Kuan GM, Lopez MM, et al. Characterization of Aedes aegypti (Diptera: Culcidae) production sites in urban Nicaragua. J Med Entomol. 2007;44:851-60.

27. Lamothe G. Adjusting the Mantel Haenszel test statistic for cluster sampling. Annex to Andersson N, Lamothe G. Clustering and meso-level variables in cross-sectional surveys: food aid during the Bosnian crisis. BMC Health Serv Res. 2011;11(supp2): doi:10.1186/s12889-017-4296-6.

28. Omar RZ, Thompson SG. Analysis of a cluster randomised trial with binary outcome data using a multi-level model. Stat Med. 2000;19:2675-88.

29. Honaker J, King G, Blackwell M. Amelia II. A program for missing data. http://gking.harvard.edu/amelia. Accessed 1 May 2017.

30. Rubin DB. Multiple imputation for non-response in surveys. New York: Wiley; 1987.

31. Imai K, King G, Lau O. Zelig: everyone's statistical software, 2007. http:// gking.harvard.edu/zelig. Accessed 1 May 2017.

32. Andersson N, Paredes S, Legorreta J, Ledogar RJ. Who pays for measles? The economic argument in favour of sustained universal child immunization. Health Policy Plan. 1992;7:352-63.

33. Andersson N, Cockcroft A, Ansari N, Omer K, Losos J, Ledogar R, et al. Household cost-benefit equations and sustainable universal childhood immunisation: a randomised cluster controlled trial in south Pakistan [ISRCTN12421731]. BMC Public Health. 2005:5:72.

34. Andersson N, Mitchell S. Epidemiological geomatics in evaluation of mine risk education in Afghanistan: introducing population weighted raster maps. Int J Health Geogr. 2006;5:1.

35. Taylor DW, Bosch EG. CTS: a clinical trials simulator. Stat Med. 1990;9:787-801.

36. Stoddard ST, Morrison AC, Vazquez-Prokopec GM, Paz Soldan V, Kochel $T J$, Kitron $U$, et al. The role of human movement in the transmission of vector-borne pathogens. PLoS Negl Trop Dis. 2009;3:e481.

37. Stoddard ST, Forshey BM, Morrison AC, Paz-Soldan VA, Vazquez-Prokopec GM, Astete $\mathrm{H}$, et al. House-to-house human movement drives dengue virus transmission. Proc Natl Acad Sci U S A. 2013;110:994-9.

38. CIET policy on security, storage and destruction of records, 2012. Available from http://www.ciet.org/_documents/Policy-records.pdf. Accessed 1 May 2017.

39. World Health Organisation. Temephos in drinking-water: Use for vector control in drinking-water sources and containers (Background document for development of WHO Guidelines for Drinking-water Quality). http://www.who.int/water_ sanitation_health/dwq/chemicals/temephos.pdf. Accessed 1 May 2017.

40. WHO. Dengue: guidelines for diagnosis, treatment, prevention and control. Geneva: World Health Organization; 2009.

41. Devine GJ, Furlong MJ. Insecticide use: contexts and ecological consequences. Agric Hum Values. 2007;24:281-306.

42. USEPA. Temephos RED (Reregistration Eligibility Decision). Washington, DC: US Environmental Protection Agency; 2008. https://www3.epa.gov/ pesticides/chem_search/reg_actions/reregistration/red_PC-059001_1-Jun-00. pdf. Accessed 1 May 2017. 
43. Anadu DI, Anaso HU, Onyeko OND. Acute toxicity of the insect larvicide Abate (temephos) on the fish Tilapia melanopleura and the dragonfly larvae Neurocordelia virginiensis. J Environ Sci Health B. 1996;31:1363-75.

44. Sparling DW, Lowe TP, Pinkney AE. Toxicity of abate to green frog tadpoles. Bull Environ Contam Toxicol. 1997;58:475-81.

45. Aiub CAF, Coelho ECA, Sodre E, Pinto LFR, Felzenszwalb I. Genotoxic evaluation of the organophosphorous pesticide temephos. Genet Mol Res. 2002;1:159-66.

46. Braga IA, Lima JBP, da Silva SS, Valle D. Aedes aegypti resistance to temephos during 2001 in several municipalities in the States of Rio de Janeiro, Sergipe, and Alagoas, Brazil. Mem. Inst. Oswaldo Cruz. Rio de Janeiro. 2004;99:199-203

47. Chen CD, Nazni WA, Lee HL, Sofian-Azirun M. Susceptibility of Aedes aegypti and Aedes albopictus to temephos in four study sites in Kuala Lumpur City Center and Selangor State. Malaysia Tropical Biomedicine. 2005;22:207-16.

48. Jirakanjanakit B, Saengtharatip S, Rongnoparut P, Duchon S, Bellec C, Yoksan $\mathrm{S}$. Trend of temephos resistance in Aedes aegypti mosquitoes in Thailand during 2003-2005. Environ Entomol. 2007;36:506-11.

49. Lima JBP, da Cunha MP, da Silva Junior RC, Galardo AKR, da Silva SS, Braga IA, et al. Resistance of Aedes aegypti to organophosphates in several municipalities in the state of Rio de Janeiro and Espö'rito Santo. Brazil Am J Trop Med Hyg. 2003;68:329-33.

50. Rodriguez MM, Bisset J, de Fernandez DM, Lauzan L, Soca A. Detection of insecticide resistance in Aedes aegypti (Diptera: Culicidae) from Cuba and Venezuela. J Med Entomol. 2001;38:623-8.

51. Rodriguez MM, Bisset JA, Fernandez D. Levels of insecticide resistance and resistance mechanisms in Aedes aegypti from some Latin American countries. J Am Mosq Control Assoc. 2007;23:420-9.

52. Brengues C. Pyrethroid and DDT cross-resistance in Aedes aegypti is correlated with novel mutations in the voltage-gated sodium channel gene. Med Vet Entomol. 2003;17:87-94.

\section{Submit your next manuscript to BioMed Central and we will help you at every step:}

- We accept pre-submission inquiries

- Our selector tool helps you to find the most relevant journal

- We provide round the clock customer support

- Convenient online submission

- Thorough peer review

- Inclusion in PubMed and all major indexing services

- Maximum visibility for your research

Submit your manuscript at www.biomedcentral.com/submit 